\title{
Geo-hazard Forecast Based on Grey Model in Mine Area
}

\author{
DING Feng ${ }^{1 \mathrm{a}}$ WANG Dongdong ${ }^{2 b}$ Hao Wenxin ${ }^{1 \mathrm{c}}$ \\ ${ }^{1}$ School of Management China university of Mining \& Technology (Beijing), PRC. \\ ${ }^{2}$ College of earth science and engineering Shandong University of Science \& Technology, PRC. \\ a461569067@qq.com, bwdd02_1@163.com, ${ }^{\mathrm{c}}$ china_zenith@qq.com.
}

Key words: geo-hazards; grey model; prediction; mine area

Abstract: The paper studied a forecast method of geo-hazards in mine area, by use of grey model, it is expected that in 2020, the loss statistics of mine geo-hazards in the area will increase steadily, the loss of water-inrush, slag, ground subsidence and landslide collapse will reach relatively 381,723 dollars, 512,759 dollars, $50.91 \mathrm{~km}^{2} / 10^{3} \mathrm{~km}^{2}, 837.88610^{4} \mathrm{~m}^{3}$. The author gave some suggestions for the sustainable development, ecological environment construction and protection.

\section{Introduction}

With the development of urban scale and construction of various projects recently, more and more geo-hazards are widespread in some area. Increasingly great threats will be imposed on people's life and property and directly affect development of national economy and stability of society.

Prevention and control of geo-hazard is turned into a hotspot issue. The mine geo-hazard is an important branch of geo-hazard, at present the mine geo-hazard has many characteristics, such as varied, widely distributed, great influence, conspicuous potential hazard. Based on statistical data, the main sorts are as follows: water-inrush, slag, ground subsidence and landslide collapse and so on.

Decision-making based on prediction. The common methods are Time-Series method, Season change method, Markov forecast method, Grey model and Delphi method. The paper adopts the grey model to forecast geo-hazard loss in future.

\section{Grey Model}

Grey model was pioneered by Deng Julong in 1982. Based on the grey system theory, a forecast model is established for grey series. GM $(1,1)$ shows a differential equation involving a function of one variable, which basic thinking is that builds dynamic or static white module based given data sequence according to a kind rule, then depends on some change or solution to calculate grey module in the future; in the grey module, find gradually dynamic variation and forecast its future development trend.

Building model step as follows:

(1) Given raw series $x^{(0)}$

$$
\mathrm{X}^{(0)}=\left\{\mathrm{x}^{(0)}(1), \mathrm{x}^{(0)}(2), \ldots \mathrm{x}^{(0)}(\mathrm{t}), \ldots \mathrm{x}^{(0)}(\mathrm{n})\right\}
$$

Meanwhile, $x^{(0)}(t)$ represents the time series, $n \geqslant 4$

(2) Based on series $X^{(0)}$, in order to overcome the defect of raw fluidity and randomcity, then generate a new sequence $X^{(1)}$, by accumulation generating operator , build a MEAN $X^{(1)}$

$$
\mathrm{X}^{(1)}=\left\{\mathrm{x}^{(1)}(1), \mathrm{x}^{(1)}(2), \ldots \mathrm{X}^{(1)}(\mathrm{t}), \ldots \mathrm{X}^{(1)}(\mathrm{n})\right\} .
$$


(3) Therefore, $x^{(1)}(k)=\sum_{t=1}^{k} x^{(0)}(t), k=1,2,3, \ldots n$

GM $(1,1)$ calculation process is as follows.

I White differential equation: $\mathrm{dx}{ }^{(1)} / \mathrm{dx}+\mathrm{ax}^{(1)}=\mathrm{u}$

Among which, a represents development coefficient, $\mathrm{u}$ represents grey function action.

II Background function action $\mathrm{ax}^{(1)}\left(\mathrm{x}^{(1)}(\mathrm{k}+1)\right)=-\mathrm{ah}^{(1)}(\mathrm{k}+1)+\mathrm{u}$

III Fundamental relations

$$
\begin{aligned}
& \mathrm{a}^{(1)}\left(\mathrm{x}^{(1)}(\mathrm{k}+1)\right)=-\mathrm{x}^{(0)}(\mathrm{k}+1) ; \mathrm{ah}^{(1)}(\mathrm{k}+1)=1 / 2\left(\mathrm{x}^{(1)}(\mathrm{k})+\mathrm{x}^{(1)}(\mathrm{k}+1)\right) \\
& \text { IV Parameter sequence } \hat{a} ; \hat{a}=[a, u]^{T}
\end{aligned}
$$

$\mathrm{V}$ By the least square method, then get an equation.

$$
\begin{array}{cc}
\hat{a}=\left(B^{T} B\right)^{-1} B^{T} y_{N} & \\
B=\left[\begin{array}{ll}
-\frac{1}{2}\left(x^{(1)}(1)+x^{(1)}(2)\right) & 1 \\
-\frac{1}{2}\left(x^{(1)}(2)+x^{(1)}(3)\right) & 1 \\
\cdots & \ldots \\
-\frac{1}{2}\left(x^{(1)}(n-1)+x^{(1)}(n)\right) & 1
\end{array}\right] & y_{N}=\left[\begin{array}{l}
x^{(0)}(2) \\
x^{(0)}(3) \\
\ldots \\
x^{(0)}(n)
\end{array}\right]
\end{array}
$$

Then, get $\operatorname{GM}(1,1)$ :

$$
\hat{x}^{(1)}(t+1)=\left(x^{(0)}(1)-u / a\right) e^{-a t}+u / a
$$

Finally building grey model, judge residual、 relevancy extent

(1)Residual includes absolute error and relative error.

$$
\begin{gathered}
\text { Absolute error: } \quad e^{(0)}(t)=x^{(0)}(t)-\hat{x}^{(0)}(t) \quad(t=1,2,3, \ldots n) \\
\text { Relative error: } \quad \pi^{(0)}(t)=\left[e^{(0)}(t) / x^{(0)}(t)\right] \quad(t=1,2,3, \ldots n)
\end{gathered}
$$

The result is acceptable, if residual is not beyond the range.

(2) Relevancy extent examination

$$
\text { if } \Delta(t)=\left|x^{(0)}(t)-\hat{x}^{(0)}(t)\right|, \text { so } \xi(t)=\frac{\operatorname{Min}\{\Delta(t)\}+\rho \operatorname{Max}\{\Delta(t)\}}{\Delta(t)+\rho \operatorname{Max}\{\Delta(t)\}},(t=1,2,3 \ldots n)
$$

Represents maximum percentage, in general $\rho$ is chosen by $50 \%$.

$$
\text { Relevancy extent: } \xi=\frac{1}{\mathrm{n}-1} \sum_{t=1}^{n} \xi(t)
$$

(3)Posteriori variance examination

Firstly, original data mean value

$$
\begin{aligned}
& x^{-(0)}=\frac{1}{n} \sum_{t=1}^{n} x^{(0)}(t) \\
& \text { variance } S_{0}=\sqrt{\frac{\sum_{t=1}^{n}\left[x^{(0)}(t)-x^{(0)}\right]^{2}}{n-1}}
\end{aligned}
$$

$$
\text { Secondly, get residual mean value } e^{e^{-(0)}}=\frac{1}{n} \sum_{t=1}^{n} e^{(0)}(t)
$$


And variance

$S_{1}=\sqrt{\frac{\sum_{t=1}^{n}\left[e^{(0)}(t)-e^{-(0)}\right]^{2}}{n-1}}$

Quotient of variance: $C=\frac{S_{1}}{S_{0}}$

Finally calculate small error probability $P . \quad P=P\left\{\left|e^{(0)}(t)-e^{-(0)}\right|<0.6745 \times S_{0}\right\}$

Judge a good model depending on $\mathrm{C}$ and $\mathrm{P}$ (Table 1).

Table 1 Forecast accuracy grade

\begin{tabular}{ccc}
\hline $\mathrm{P}$ & $\mathrm{C}$ & Forecasting Accuracy Grade \\
\hline$>0.95$ & $<0.35$ & Excellence \\
$>0.80$ & $<0.50$ & Eligibility \\
$>0.70$ & $<0.65$ & Reluctant eligibility \\
$\leq 0.70$ & $\geq 0.65$ & Disqualification \\
\hline
\end{tabular}

\section{Application of Grey Model}

According to the above analysis of these in A mine of Youyu County, Shanxi Province during last six years, the output data have been chosen as the original data sequence Since 2009, and GM(1,1) adopted as the grey model, Time-response functions of water-inrush, slag, ground subsidence, landslide \& collapse are obtained relatively as follows:

$$
\begin{aligned}
& \hat{x}^{(1)}(t+1)=167931.0622 e^{-0.0746 t}-161959.8858 \\
& \hat{x}^{(1)}(t+1)=216628.2730 e^{-0.0783 t}-209283.1799 \\
& \hat{x}^{(1)}(t+1)=16.9624 e^{-0.0999 t}-16.0354 \\
& \hat{x}^{(1)}(t+1)=550.5834 e^{-0.0382 t}-541.1410
\end{aligned}
$$

The average relative error of water-inrush, slag, ground subsidence, landslide \& collapse: $1.2518 \%$, $1.3657 \%, 0.8066 \%, 0.5460 \%$.

\section{Model Accuracy Examination}

The prediction result is more accuracy. $(\mathrm{P}=0.97>0.95(\mathrm{Ex}))$.

\section{Prediction Results Evaluation}

According to table 2, by analysis of model reduction value and residual, it is feasible that predictive results of loss statistics of mine geo-hazards, high forecasting accuracy by use of the grey model GM $(1,1)$. The mine geo-hazards will increase stably in the next 6 years will be shown in table 3 .

\section{The Problems in Mine Hazards-inducing}

(1) Natural conditions, including geological factors; terrain conditions; climatic conditions; terrestrial hydrological and so on, play an important role in inducing hazards. 
(2) Socio-economic conditions, including population density; property type and value of density; use of resources and the environment; hazard prevention and so on.

Table 2 Loss Statistics of mine geo-hazards ${ }^{[3]}$

\begin{tabular}{ccccc}
\hline Time(Year & Water-inrush $(\$)$ & $\begin{array}{c}\text { Slag } \\
(\$)\end{array}$ & $\begin{array}{c}\text { Ground } \\
\text { subsidence } \\
\left(\mathrm{km}^{2} / 10^{3} \mathrm{~km}^{2}\right)\end{array}$ & $\begin{array}{c}\text { Landslide \& } \\
\text { collapse }\left(10^{4} \mathrm{~m}^{3}\right)\end{array}$ \\
\hline 2009 & 165000 & 203500 & 17.90 & 524.40 \\
2010 & 183000 & 228500 & 18.50 & 575.60 \\
2011 & 196000 & 257000 & 20.80 & 595.30 \\
2012 & 208000 & 279600 & 23.20 & 610.10 \\
2013 & 222000 & 296000 & 25.40 & 640.50 \\
2014 & 248000 & 318000 & 27.80 & 670.40 \\
\hline
\end{tabular}

Table 3 Prediction results of Loss Statistics of mine geo-hazards ${ }^{[3]}$

\begin{tabular}{ccccc}
\hline Time(Year) & $\begin{array}{c}\text { Water- } \\
\text { inrush }(\$)\end{array}$ & $\begin{array}{c}\text { Slag } \\
(\$)\end{array}$ & $\begin{array}{c}\text { Ground } \\
\text { subsidence } \\
\left(\mathrm{km}^{2} / 10^{3} \mathrm{~km}^{2}\right)\end{array}$ & $\begin{array}{c}\text { Landslide \& } \\
\text { collapse }\left(10^{4} \mathrm{~m}^{3}\right)\end{array}$ \\
\hline 2015 & 262814 & 346596 & 30.89 & 692.298 \\
2016 & 283184 & 374836 & 34.14 & 719.236 \\
2017 & 305133 & 405377 & 37.72 & 747.222 \\
2018 & 328782 & 438406 & 41.69 & 776.298 \\
2019 & 354265 & 474127 & 46.07 & 806.504 \\
2020 & 381723 & 512759 & 50.91 & 837.886 \\
\hline
\end{tabular}

\section{Suggestions and Solutions}

According to the threat of mine geo-hazards, solutions are as follows:

(1) Strengthen surveying and clarify distribution \& formation laws of geo-hazards.

(2)Prevent and control geological environment, and diminish mine hazard-inducing factors.

(3)Build information system of mine geo-hazards, and perfect monitoring and warning system.

(4)Enact laws of mine geo-hazards prevention and reduction.

\section{References.}

[1] DENG Julong. Grey forecasting and decision-making, Huazhong University of science and Technology Press, Yu Jiashan, Wuchang 1986.

[2] DING Feng, WANG Lijie. Risk Management Assessment in Landslide in Dashikou of Ying County, Shanxi Province. Advanced Materials Research Vols. 864-867 (2014) pp 1116-1120.

[3] D.Feng.Geological Hazards Mechanism and Risk Assessment in Pinglu District \& Shuocheng District.PhD Dissertation.Beijing,China University of Mining\&technology,2012.(In Chinese) 